\title{
Dispersive Delay Lines Based on the Use Of Narrow Open Metal Reflectors and Fan-Shaped Transducers
}

\author{
K.C. Lee ${ }^{1}$, V. P. Plessky², S. M. Balashov ${ }^{3}$, C.W. Nam ${ }^{4,+}$, and C.U. Kim ${ }^{1,4}$
}

\begin{abstract}
An in-line dispersive delay line (DDL) demands low and controllable reflectivity of the reflectors, especially if the surface acoustic wave (SAW) propagates all the way along the reflecting structure. The metal reflectors are usually too strong and introduce too much attenuation in such a device. The proposed solution of this problem is to spatially separate the acoustic channels for different frequencies with the help of Fan-shaped Transducers (FIDT) and to use narrow open metal reflectors to reduce reflectivity. Special arrangement of FIDT is performed to use 180-deg. reflection of the SAW. Narrow open metal strips with a metallisation ratio of the order of $20 \%$ are used as reflectors with small and controllable reflectivity. Reflectivity of such strips is estimated both theoretically and experimentally. Experimental performance of the proposed DDL is presented.
\end{abstract}

Keywords : SAW, FIDT, Reflector, DDL

\section{INTRODUCTION}

In-line Dispersive Delay Lines (DDL) [1] normally use shallow grooves with controlled reflectivity. For metal reflectors the metal dots were used by many authors (see e.g. [2]) to obtain controllable weak reflectivity. Development of SAW device technology during the last 15 years allows us to now re-visit this area and to use narrow open electrodes with the metallization ratio $\mathrm{m} / \mathrm{p}<0.2$ as reflectors. The advantage of open electrodes is that the resistive losses are negligible and the reflectivity can be easily controlled by the electrode width. In this paper the reflectivity of narrow electrodes on $\mathrm{YZ}^{-\mathrm{LiNbO}_{3} \text { is }}$ modelled, using FEM/BEM software, and obtained from the measurements of the test devices. We discuss the possibility of using FIDT in a "mutually-blind" combination of transducers for the design of DDL and show the experimental results of the study regarding such structures.

\footnotetext{
${ }^{1}$ University of Ulsan, Ulsan, South Korea.

${ }^{2}$ GVR Trade SA, Bevaix, Switzerland,

${ }^{3}$ SAWDES Ltd, Campinas, San-Paulo, Brazil,

${ }^{4}$ EFTECH, Ochang, South Korea

${ }^{+}$Corresponding Author :cwnam@mail.ulsan.ac.kr

(Received : Sep. 12, 2012, Accepted : Oct. 27, 2012)
}

This is an Open Access article distributed under the terms of the Creative Commons Attribution Non-Commercial License(http://creativecommons.org/licenses/by$\mathrm{nc} / 3.0$ )which permits unrestricted non-commercial use, distribution, and reproduction in any medium, provided the original work is properly cited.

\section{REFLECTIVE PROPERTIES OF NARROW ELECTRODES}

Although some data on the reflectivity of $\mathrm{Al}$ electrodes

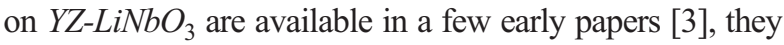
are fragmentary and difficult to use. However, it is evident that with the decrease of the width of open electrodes its reflectivity also decreases and that the reflection coefficient for a fraction of $1 \%$ can be easily reached.

For modelling with FEMSAW software of narrow reflectors we used the "test structure" including 3 IDTs. The structure consists of launching an IDT with 5 periods and a reflector under the test with 5 open electrodes in the middle between two short probing IDTs, having one period each. Split finger geometry is used for IDTs to minimize reflections. Separation of launching and probing IDTs allows one to "see" in the time domain both incident and reflected pulses. In the Y-parameter regime all transducers, except launching have zero voltage - so, there is no regeneration of SAW or absorption of energy. Comparing reflected signals registered on one of the probing IDTs in the time domain with transmitted signals on another probing IDT, one can find the reflection coefficient. The results of such a simulation are shown in Fig. 1. The value $\mathrm{h} / \lambda=2.5 \%$ (h-is metal thickness and $\lambda$ - is Bragg frequency) was chosen for simulations. 


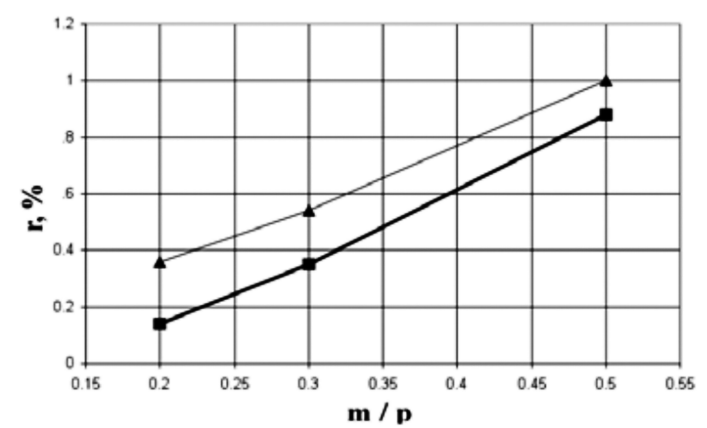

Fig. 1. Reflectivity per finger as a function of $\mathrm{m} / \mathrm{p}$. Here, $\mathrm{m}$ is electrode width, $\mathrm{p}$ - is the period of reflectors. The thin curve with points marked as triangles is the simulation result and the thick curve with points marked as squares is the experimental result.

Simulation curve can be approximated by the straight line

$$
r(\%)=2.17(\mathrm{~m} / \mathrm{p})-0.087
$$

For experiments aimed to determine the reflectivity of narrow fingers we used the structure with 2 IDTs and a reflector symmetrically situated between them. The following algorithm was used: Reflection coefficient $\mathrm{S}_{11}$ was transformed to the time-domain and the contribution, which corresponds to the propagation (Input IDT)(reflectors)-(Back to Input IDT), was extracted from this response and transformed back into the frequency domain. The resulting $\mathrm{S}_{11}$ contains the contribution of the following three processes: 1)-SAW generation by IDT; 2) - Reflection of the SAW from reflectors; 3) Absorption of reflected SAW with the same IDT. Assuming that $\mathrm{S}_{12}$ contains only two parts of the same process, namely part 1 and 3, extraction of the resulting $S_{11}$ from $S_{12}$ provides the total reflectivity of reflectors under study. The result of use of such an algorithm is shown in Fig. 1 for $h /=1.73 \%(h=3000$ A, $p=8.66 \mathrm{~m})$. Considering the difference of thickness in regards to the simulation and experimental result it could be concluded that reasonable agreement between the simulation and experiment exists and (which is important for construction of DDL) a metal reflector with small reflectivity could be easily obtained.

\section{EFFECTIVE APERTURE OF FIDT}

In this chapter we consider the properties of FIDT important for its use in DDL. It is well known that FIDT irradiates SAW with only a fraction of its total aperture, which we will call Local Aperture (LA). To derive the formula for the LA, consider the case of linear FIDT, i.e. the IDT with the monotonic change of the local wavelength with the change of vertical coordinate $\mathbf{Y}$ (Fig. 2). In this case, the curvature of the fingers for the entire IDT could be characterized by the single angle of inclination of the border finger $\mathbf{q}$ (see Fig. 2). Analyzing Fig. 2 for the $\mathbf{q}$ one has

$$
\tan (\vartheta)=\frac{N}{W}\left(\lambda_{\text {max }}-\lambda_{\text {min }}\right)=\frac{N V_{r} B}{W f_{\text {min }} f_{\max }}
$$

Here, $\mathbf{f}_{\min }\left(\mathbf{f}_{\max }\right)$ is frequency, which corresponds to $\lambda_{\max }$ $\left(\lambda_{\text {min }}\right) . \mathbf{V}_{\mathrm{r}^{-}}$is the SAW velocity, $\mathbf{N}$ - is the number of periods and $\mathbf{B}$ - is bandwidth of the FIDT. In case of linear FIDT

$$
\begin{aligned}
& f_{\text {min }}=f_{o}-\frac{B}{2} ; \\
& f_{\text {max }}=f_{0}+\frac{B}{2} .
\end{aligned}
$$

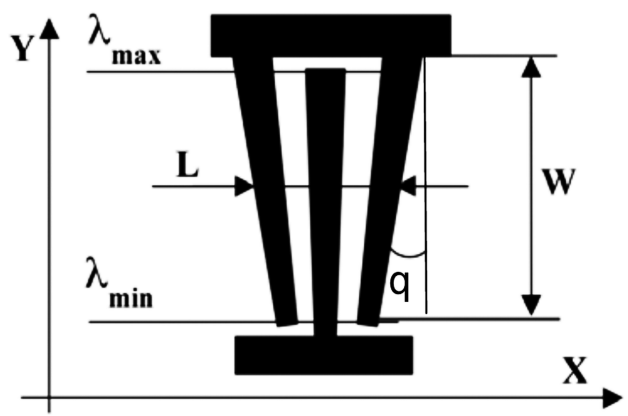

Fig. 2. Geometry of linear FAN IDT. $1_{\max }\left(1_{\min }\right)$ is the maximum (minimum) wavelength of the FIDT. L - is the length at the level, which corresponds to the central frequency of the FIDT

where $\mathbf{f}_{0}$ is the central frequency of the FIDT. Substituting (2) into (1), one has

$$
\tan (\vartheta)=\frac{N V_{r} B}{W\left(f_{0}^{2}-\frac{B^{2}}{4}\right)} \approx \frac{N V_{r} B}{W f_{0}^{2}}\left(1+\alpha^{2}\right)
$$

where for the last equation the small parameter

$$
\alpha=\frac{\mathrm{B}}{2 \mathrm{f}_{0}}<<1
$$

was introduced. For small qzero order approximation gives 


$$
\tan (\vartheta) \approx \vartheta=\frac{N V_{r} B}{W f_{0}^{2}}=\frac{N \lambda_{0} B}{W f_{0}}=\frac{L B}{W f_{0}}
$$

where $\lambda_{0}$ is the wavelength, corresponding to central frequency $\mathbf{f}_{0}$ of the IDT.

Consider now the effective aperture. For any frequency of FAN IDT generated inside its passband

$$
P=\frac{f}{N} ; \quad\left(f_{\min } \leq f \leq f_{\max }\right)
$$

Here, $\mathrm{P}$ is $3-\mathrm{dB}$ bandwidth. For simplicity we used the formula for non-apodized IDT. Due to the fact that we have linear FIDT, the proportion between apertures and local frequencies can be expressed into the following form

$$
\frac{\mathrm{D}}{\mathrm{W}}=\frac{\mathrm{P}}{\mathrm{B}}
$$

where $\mathrm{D}$ is the effective aperture, which corresponds to the given frequency of the SAW generation. Substituting (4) into (5), one has

$$
D=W \frac{P}{B}=\frac{W f}{N B}
$$

Using (3), we have

$$
\mathrm{D}=\frac{\mathrm{fV}_{\mathrm{r}}}{\tan (\vartheta)\left(\mathrm{f}_{0}^{2}-\alpha^{2}\right)} \approx \frac{\mathrm{V}_{\mathrm{r}}}{\vartheta \mathrm{f}_{0}\left(1-\alpha^{2}\right)} \frac{\mathrm{f}}{\mathrm{f}_{0}}=\frac{\mathrm{V}_{\mathrm{r}}}{\vartheta \mathrm{f}_{0}\left(1-\alpha^{2}\right)} \frac{\lambda_{0}}{\lambda}
$$

Note that

$$
\frac{f}{f_{0}}=\frac{f_{0}+\Delta f}{f_{0}}=1+\frac{\Delta f}{f_{0}}
$$

where

$$
-\frac{B}{2} \leq \Delta f \leq \frac{B}{2}
$$

It gives the following estimation

$$
\left|\frac{\Delta \mathrm{f}}{\mathrm{f}_{0}}\right| \leq \frac{\mathrm{B}}{2 \mathrm{f}_{0}}=\alpha
$$

Thus, for an effective aperture in the zero order approximation one has

$$
\mathrm{D}=\frac{\mathrm{V}_{\mathrm{r}}}{\vartheta \mathrm{f}_{0}}=\frac{\lambda_{0}}{\vartheta}
$$

The result obtained could be used for evaluation of the aperture of reflectors (it should be equal to the LA of incident SAW) in case of use of FIDTs for construction of the DDL.

\section{IN-LINE DDL DESIGN}

Geometry of the designed DDL is shown in Fig. 3. It consists of two acoustic channels with Input and Output FAN IDTs placed in each of them. Input and output IDTs from different channels are connected as it is shown in Fig. 3. Two Input IDTs, being connected in series, generate in both channels the SAW with the same wave front. The Output IDTs are connected with a $180^{\circ}$ phase shift. For such geometry SAW directly propagating from Input to Output IDTs will not generate any response in Output IDT, because the signal from the first channel will cancel the signal from the second one. Note that the condition of signal cancellation is valid for any frequency of the SAW. The SAW, which passes under the Output IDT, reaches the arrays of reflectors in each channel. The aperture of each reflector corresponds to the effective aperture $\mathrm{D}$ of the FIDT for a given frequency and the centre of the effective aperture coincides with the centre of the region of effective reflection for a given SAW by reflective arrays. Because the reflectors in the second channel are displaced by quarter of local resonance wavelength each, the reflected SAW in the second channel has $180^{\circ}$ shift comparable to reflected SAW in the first channel. Due to this additional shift, the reflected SAW will be summarised constructively in Output IDT, producing an output signal. Note that this geometry contains two potentially beneficial features. First - the phase correction stripe could be inserted between the Output IDT and reflectors, because FIDT separates the SAWs with different frequencies along the aperture. Second - the number of reflectors, which SAW crosses, is much less than the total number of reflectors, which reduces the second order effects influence.

DDLs have the following parameters: Aperture of each channel was chosen to be $1.2 \mathrm{~mm}$. Processing time of DDL is $\mathbf{T}=\mathbf{1 0} \mathbf{~ m s}, \mathbf{B}=\mathbf{2 4} \mathrm{MHz}, \mathbf{f}_{0}=\mathbf{2 0 0} \mathrm{MHz}$. One of the DDLs had ordinary IDTs with Number of periods equal to 5.5 (Type 1). The second DDL had FIDTs with 45 periods and all parameters of DDL equal to the previous one (Type 2), which gives $\mathrm{q}=2.3 \mathrm{deg}$, and $\mathrm{D}=426 \mathrm{~mm}\left(\mathbf{V}_{\mathrm{r}}=\right.$ $3421 \mathrm{~m} / \mathrm{s}$ ). Aperture of reflectors for the DDL of Type 2 was equal to $\mathrm{D}$. 


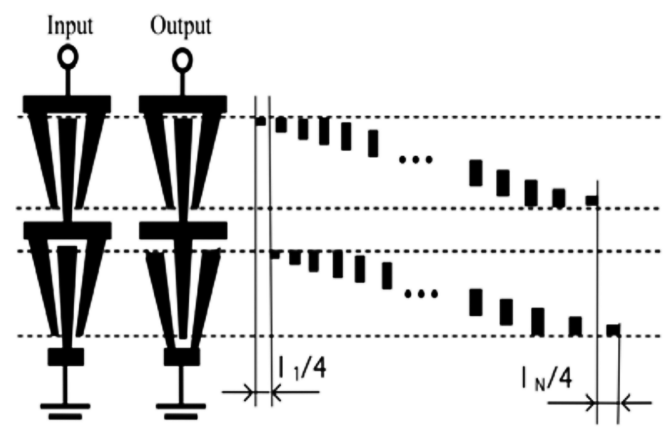

Fig. 3. Geometry of the DDL. Two acoustic channels are highlighted by dashed lines. $1_{i}$ - are the wavelengths, which correspond to the frequency of local synchronism.

\section{EXPERIMENTAL RESULTS}

Gated frequency response of DDL of Type 1 is shown on Fig. 4 and correspondent group delay on Fig. 5.

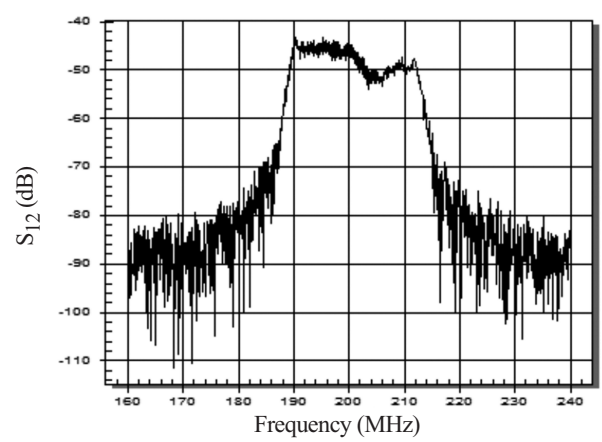

Fig. 4. Frequency response of the DDL of Type 1.

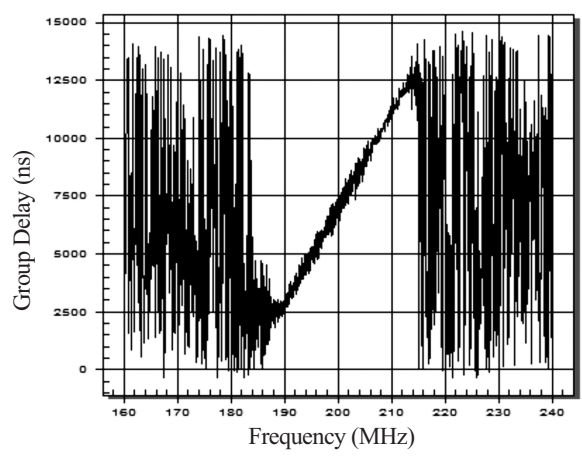

Fig. 5. Group Delay for the DDL of Type 1.

Phase deviation from ideal quadratic function for this case was $+/-25^{\circ}$. High frequency degradation of the passband was due to the contribution of bulk wave generation. For DDL of Type 2 the same parameters are shown in Fig. 6 and Fig. 7.

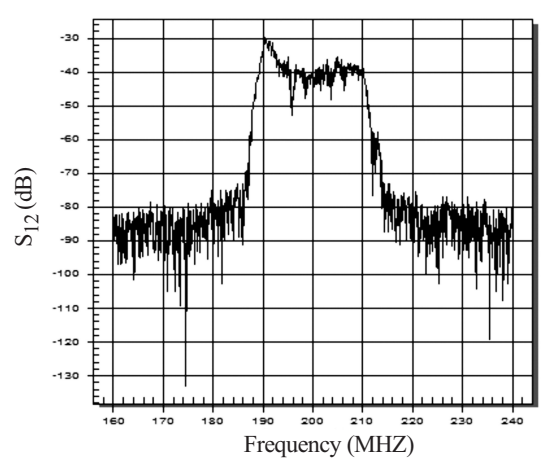

Fig. 6. Frequency response of the DDL of Type 2.

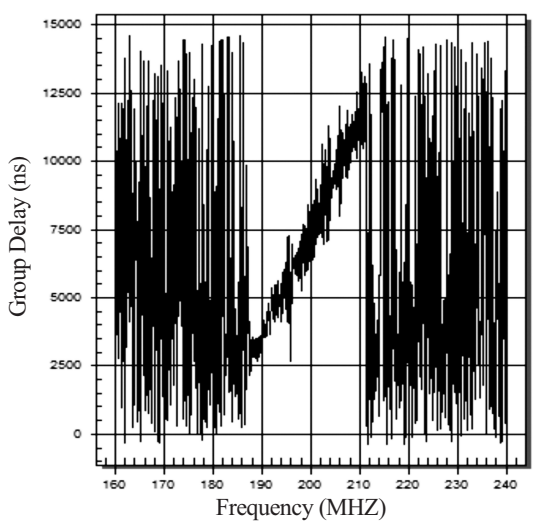

Fig. 7. Group Delay for the DDL of Type 2.

For the second DDL ripples appeared to be bigger. The nature of these ripples is currently under investigation.

\section{CONCLUSIONS}

It was shown that FIDTs and narrow metal reflectors could be used as the elements for the construction of DDLs. We used narrow open metal reflectors to reduce reflectivity. It was also theoretically and experimentally shown that metal reflectors with fan shape structures could separate SAW signals with different frequencies.

\section{ACKNOWLEDGEMENT}

This work was supported by the 2009 Research Fund of the University of Ulsan.

\section{REFERENCES}


[1] R.E. Chapman, R.K. Chapman, D.P. Morgan, and E.G.S. Paige, "In-line reflective array devices", Proc. IEEE Ultrason. Symp., pp. 728-733, 1978.

[2] R. C. Woods, "Dispersive delay lines using $180^{\circ}$ reflecting metal dot arrays", Proc. IEEE Ultrason.
Symp., pp. 88-91, 1982.

[3] D. Chen and H. A. Haus, "Analysis of metal-strip SAW gratings and transducers", IEEE Trans. Sonics Ultrason., Vol. SU-32, pp. 395-408, 1985.

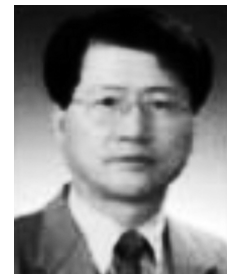

Kyu-Chol Lee received his Ph.D in 1981 in department of electrical engineering from Yonsei University, Kora. He is currently a professor in Ulsan university.

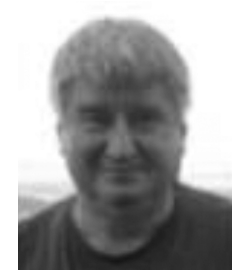

Plessky Victor received $\mathrm{PhD}$ degree from Moscow Physical-Technical (FizTech) University in a year 1978 and Dr. es Scie. degree in 1987 from Russian Acad. of Sciences. Full Professor title from Russian Government in a year 1995. Currently Dr. V. Plessky is CEO and owner of consulting company GVR Trade SA. His sphere of interests is micro- and nanoacoustics, SAW devices, sensors, SAW-tags.

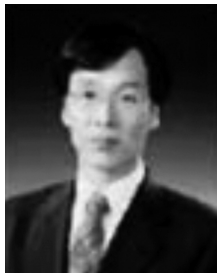

Chang-Woo Nam received his Ph.D in 1994 in department of electrical engineering from the Pennsylvania state university, USA. He is currently a professor in Ulsan university

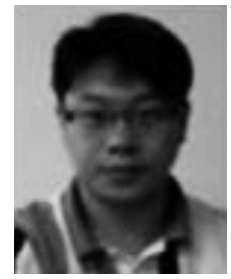

Che-Uk Kim received His M.S in 2001 in department of electrical engineering from Ulsan university, korea. He is currently working on EFTECH, Korea 